Preprint typeset in JHEP style - HYPER VERSION

HUTP-02/A051

\title{
Effective Field Theory for Massive Gravitons and Gravity in Theory Space
}

\author{
Nima Arkani-Hamed, Howard Georgi and Matthew D. Schwartz \\ Jefferson Laboratory of Physics, Harvard University, Cambridge, MA 02138 \\ email: arkani@carnot.harvard.edu, \\ georgi@physics.harvard.edu, matthew@feynman.princeton.edu
}

\begin{abstract}
We introduce a technique for restoring general coordinate invariance into theories where it is explicitly broken. This is the analog for gravity of the Callan-Coleman-WessZumino formalism for gauge theories. We use this to elucidate the properties of interacting massless and massive gravitons. For a single graviton with a Planck scale $M_{\mathrm{Pl}}$ and a mass $m_{g}$, we find that there is a sensible effective field theory which is valid up to a high-energy cutoff $\Lambda$ parametrically above $m_{g}$. Our methods allow for a transparent understanding of the many peculiarities associated with massive gravitons, among them the need for the Fierz-Pauli form of the Lagrangian, the presence or absence of the van Dam-Veltman-Zakharov discontinuity in general backgrounds, and the onset of non-linear effects and the breakdown of the effective theory at large distances from heavy sources. The natural sizes of all non-linear corrections beyond the Fierz-Pauli term are easily determined. The cutoff scales as $\Lambda \sim\left(m_{g}^{4} M_{\mathrm{Pl}}\right)^{1 / 5}$ for the Fierz-Pauli theory, but can be raised to $\Lambda \sim\left(m_{g}^{2} M_{\mathrm{Pl}}\right)^{1 / 3}$ in certain non-linear extensions. Having established that these models make sense as effective theories, there are a number of new avenues for exploration, including model building with gravity in theory space and constructing gravitational dimensions.
\end{abstract}




\section{Introduction}

It has recently been realized that non-gravitational extra dimensions can be generated dynamically from fundamentally four-dimensional gauge theories [1-3]. These theories can be represented by a graph or "theory space" consisting of sites and links [4]. In some cases, at low energies the link fields become non-linear sigma model Goldstone fields, which are eaten to yield a spectrum of massless and massive gauge bosons. This spectrum may match the Kaluza-Klein tower of a compactified higher-dimensional theory and be phenomenologically indistinguishable from an extra dimension. More interestingly, theory space generalizes the notion of higher-dimensional locality. This has allowed for the construction of purely fourdimensional models that reproduce apparently higher-dimensional mechanisms in a simple context. It has also produced powerful new tools for physics beyond the standard model with no higher-dimensional interpretation whatsoever.

So far, gravity has been added to these theories simply by minimally coupling fourdimensional Einstein gravity to the four-dimensional fields. This is a consistent thing to do, but then gravitational interactions do not respect locality in theory space. In order to have gravitational interactions be local, we would like to make gravity propagate in theory space. This is what we consider in this paper. We should emphasize that we are only interested in a low-energy description with sites and Goldstone link fields, which in a unitary gauge reproduces a finite spectrum of massless and massive gravitons. This is not "deconstruction", in the sense that we are not, for the moment, interested in a full UV completion of these theories. Instead, we are only trying to make sense of gravity living in discrete spaces, and will be content with understanding the structure of the low-energy effective theory describing such a scenario.

If we try to put gravity into theory space following the gauge theory example, we have no trouble at the level of the sites. We can easily endow each site with its own metric and general coordinate invariance symmetry. The difficulty arises when we try to write down the analog of the Goldstone boson link fields which must transform non-trivially under two general coordinate invariances. For instance, a four-dimensional field can only depend on one set of coordinates, not two, so how can it transform under two general coordinate invariances? Moreover, in a unitary gauge where the links are eaten, we have a massless diagonal graviton and a finite tower of massive gravitons. Such theories have many strange properties, and there are doubts in the literature about whether they are consistent [5-9].

In this paper, we show that there actually is a very natural way of introducing link fields. It allows us to show that the theory of a massless graviton coupled to a finite number of massive gravitons makes sense as a consistent effective field theory valid up to energies parametrically above the particle masses. The construction of the Goldstone link fields for gravity is easiest to understand in analogy with the gauge theory case which we review in detail. For example, right multiplication by an element of a gauge group in gauge theory translates to composition

with a coordinate transformation in the gravity case. This allows us to define links, with the quantum numbers of four-dimensional vectors. These links have simple transformation 
laws under pairs of general coordinate invariances, and allow the construction of interacting Lagrangians with multiple general coordinate invariances.

After describing the general formalism, we study the case of a single graviton of mass $m_{g}$ in detail. This can be understood as a two-site model in the limit where the Planck scale on one of the sites is taken to infinity. Just as for massive gauge theories, the Goldstone description is extremely useful in understanding the properties of the longitudinal polarizations of the graviton. Massive gravitons are well-known to have a number of strange properties. The mass term must have a specific Fierz-Pauli structure [10,11]. The propagator around flat space suffers from the famous van Dam-Veltman-Zakharov (vDVZ) discontinuity [12,13], though this disappears in anti-de Sitter (AdS) backgrounds [14-18]. Finally, there is the observation first made Vainshtein [19] and further explored recently in [20-22], that the gravitational field outside of a massive object breaks down at a peculiar macroscopic distance scale much larger than the naive gravitational radius. We will understand all of these properties in a transparent way, and see that they are different reflections of a single underlying cause: the scalar longitudinal mode of the graviton becomes strongly coupled at far lower energies than naively expected. Nevertheless, we will see that we have a sensible effective field theory valid up to a cutoff $\Lambda$ parametrically higher than $m_{g}$, with $\Lambda \sim\left(m_{g}^{4} M_{\mathrm{Pl}}\right)^{1 / 5}$ for the Fierz-Pauli theory. This cutoff can be pushed up to $\Lambda \sim\left(m_{g}^{2} M_{\mathrm{Pl}}\right)^{1 / 3}$ in certain non-linear extensions of the Fierz-Pauli Lagrangian. We will also be able to determine, by simple power-counting, the form of all the corrections to the Fierz-Pauli Lagrangian which are generated radiatively.

With the assurance that effective theories with multiple interacting spin two fields make sense, many potential applications can be envisioned. The most obvious application is to building gravitational extra dimensions, which we will discuss in an upcoming paper. We will conclude this paper by discussing a number of other possible applications, as well as open problems and other directions for future research along these lines.

\section{Review of gauge theory}

In this section we review some aspects of the effective theory of massive spin one fields. We emphasize advantages of the effective field theory formalism [23,24] and show why introducing a "fake" gauge invariance can be a very useful thing to do.

Consider for definiteness an $S U(n) \times S U(n)$ gauge theory, which is broken to the diagonal subgroup. At energies beneath the Higgsing scale, the theory consists only of a massive and massless gauge multiplet, or equivalently of two gauge multiplets together with the Goldstone bosons which are eaten to make the massive gauge boson heavy. In theory space language, we have a two-site model with gauge symmetry $S U(n)_{1} \times S U(n)_{2}$, with a single bi-fundamental non-linear sigma model link field $U$ :

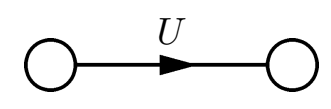


$U$ transforms linearly under the gauge symmetries as $U \rightarrow g_{2}^{-1} U g_{1}$ and induces non-linear transformations on the Goldstone boson fields when we expand $U=e^{i \pi}$. The Lagrangian can be written as

$$
\mathcal{L}=-\frac{1}{g_{1}^{2}} \operatorname{tr} F_{1}^{2}-\frac{1}{g_{2}^{2}} \operatorname{tr} F_{2}^{2}+f^{2} \operatorname{tr}\left|D_{\mu} U\right|^{2}+\cdots
$$

where $D_{\mu} U=\partial_{\mu} U+i A_{1 \mu} U-i U A_{2 \mu}$ is the covariant derivative. In the unitary gauge, we can set $U=1$ and the Lagrangian becomes

$$
\mathcal{L}=-\frac{1}{g_{1}^{2}} \operatorname{tr} F_{1}^{2}-\frac{1}{g_{2}^{2}} \operatorname{tr} F_{2}^{2}+f^{2} \operatorname{tr}\left(A_{1}-A_{2}\right)^{2}+\cdots
$$

In the limit as we take, say, $g_{1} \rightarrow 0$, the surviving massless gauge boson becomes all $A_{1}$ and completely decouples, and we are left with a single massive gauge boson $A \equiv A_{2}$. In this limit, the Lagrangian is simply

$$
\mathcal{L}=-\frac{1}{g^{2}} \operatorname{tr} F^{2}+f^{2} \operatorname{tr}\left|D_{\mu} U\right|^{2}+\cdots
$$

which in unitary gauge becomes

$$
\mathcal{L}=-\frac{1}{g^{2}} \operatorname{tr} F^{2}+f^{2} \operatorname{tr} A^{2}+\cdots
$$

So we can identify the mass of the gauge boson as $m_{A}=g f$.

Now, the physics described by the the Lagrangian with the Goldstone bosons included is identical to the unitary gauge Lagrangian without the Goldstone fields. The unitary gauge Lagrangian (2.5) does not have any gauge invariance, while the Goldstone boson Lagrangian (2.4) does. It is clear that this symmetry is a complete fake; we can always go to the unitary gauge where it is not there! This is always true for local symmetries - they are not symmetries but redundancies of description. If a theory is not gauge invariant, we can introduce Goldstone fields with appropriate transformation properties to make it gauge invariant.

However, there are important advantages to introducing the Goldstone bosons: at energies far above $m_{A}$, the Goldstones $(\pi)$ become the longitudinal component of the massive gauge boson $\left(A^{L}\right)$ :

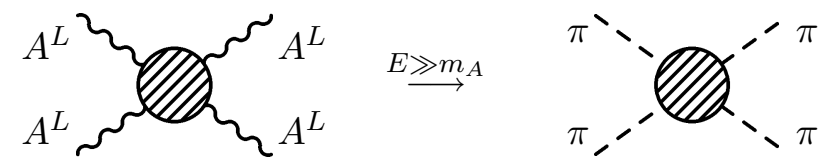

From the Goldstone description it is obvious that the interactions of these longitudinal modes become strongly coupled at a scale $\sim 4 \pi f \sim 4 \pi m_{A} / g$, since this is dimensionful scale that appears in the non-renormalizable, non-linear sigma model. Since the physics is exactly that of the unitary gauge theory, this could also have been inferred directly in unitary gauge, though the analysis would be more cumbersome and less illuminating. For instance, we can evaluate the Feynman diagrams for tree-level longitudinal gauge boson scattering. Since the polarization vector for the longitudinal gauge boson is $\epsilon_{\mu} \sim k_{\mu} / m_{A}$ at high energy, there is a danger that these amplitudes can become large. The tree-level amplitude for $A^{L} A^{L} \rightarrow A^{L} A^{L}$ 
could grow as rapidly as $g^{2}\left(E / m_{A}\right)^{4}$ from this consideration, since there are four polarization vectors. However, there is a cancellation between the direct 4-point gauge interaction and exchange diagrams for this process, and the amplitude only grows as $\sim g^{2}\left(E / m_{A}\right)^{2}$, which becomes strongly coupled at $\sim 4 \pi m_{A} / g \sim 4 \pi f$. This example illustrates why the Goldstone boson formalism is so powerful: it focuses on precisely the degrees of freedom that limit the regime of validity of the effective theory by becoming strongly coupled. These degrees of freedom are obscured in the unitary gauge.

The Goldstone description also allows us to determine the structure of the higher-order terms in the effective Lagrangian by simple power-counting. We expect at quantum level that in addition to the leading two-derivative terms in the non-linear sigma model, we generate higher derivative terms such as

$$
\sim \frac{1}{16 \pi^{2}} \operatorname{tr}\left|D_{\mu} U\right|^{4}, \frac{1}{16 \pi^{2}} \operatorname{tr}\left|D^{2} U\right|^{2}, \cdots
$$

In unitary gauge these correspond to operators of the form

$$
\frac{1}{16 \pi^{2}} \operatorname{tr} A^{4}, \frac{1}{16 \pi^{2}} \operatorname{tr}(\partial A)^{2}, \cdots
$$

However, the natural size for these operators would have been hard to understand directly in unitary gauge. These operators all explicitly break gauge invariance. How would we know how to organize the various terms that break gauge invariance? Why, for instance, since the theory is not gauge invariant, do we still use the gauge invariant $F_{\mu \nu}^{2}$ kinetic term but only break gauge invariance through the mass term? Normally, this is justified because a general kinetic term gives rise to ghosts in the propagator. But surely we must expect that non-gauge invariant kinetic terms are generated. The standard power-counting analysis shows that non-gauge invariant kinetic terms $\sim(\partial A)^{2}$ are generated, as well as non-gauge invariant $A^{4}$ type interactions. But these are down by weak-coupling factors $\sim g^{2} /\left(16 \pi^{2}\right)$ relative to the leading terms, as long as the theory is treated as an effective theory with cutoff $\sim 4 \pi m_{A} / g$. These sizes insure that all the dangerous effects associated with the nongauge invariant propagators, such as the appearance of ghosts, are deferred to the cutoff, parametrically above $m_{A}$. Furthermore, it is easy to determine the natural size of all other gauge-violating operators effects in a systematic way.

Thus, while the Goldstone description is physically identical to the unitary gauge description of a massive gauge boson, it is vastly more powerful in elucidating the physics. The Goldstone description makes it clear that the theory of a massive gauge boson is sensible up to a cutoff $\sim 4 \pi m_{A} / g$. Above this scale, an ultraviolet completion is needed. This is actually where the Goldstone description gains its full power, since finding a UV completion only necessitates finding a good UV theory that leads to the Goldstones at low energies. This can be done straightforwardly, for instance via linear sigma models (as in the standard Higgs mechanism), or QCD-like completions (as in technicolor). But none of these UV completions could have easily been guessed from the unitary gauge picture. 
Summarizing, the Goldstone boson description offers three advantages to thinking about theories with massive gauge bosons: (1) It transparently encodes the interactions of the longitudinal components of the gauge bosons at high energy, and determines the cutoff of the effective theory. (2) Simple power-counting determines the natural size of all non-gauge invariant operators. (3) It helps point the way, from the bottom-up, to possible UV completions of the physics.

\section{Building blocks for gravity in theory space}

We would like to do the same kind of analysis for gravity. We will begin with the building blocks for a general theory with many "sites" endowed with different 4D general covariances. We will show how to define "link" fields with suitable non-linear transformation properties, in analogy with the gauge theory case. We will also discuss the gravitational analog of "plaquette" operators, needed to realize higher-dimensional theory spaces.

\subsection{Sites and Links}

We start with a collection of sites $j$ each of which has its own general coordinate invariance symmetry $\mathrm{GC}_{j}$. We will label a given set of coordinates on the site $j$ with $x_{j}$. The $\mathrm{GC}_{j}$ symmetry is generated by $x_{j}^{\mu} \rightarrow f_{j}^{\mu}\left(x_{j}\right)$, where we assume the functions $f_{j}$ are smooth and invertible. In this paper we will only be concerned with local physics, and therefore ignore any issues relating to the global topology of the sites.

We would now like to introduce link fields that allow us to compare objects on different sites, which transform under different GC symmetries. In order to do this in a transparent way completely parallel to the gauge theory example, let us discuss transformation properties under GC symmetries with a simple notation. A field $\phi(x)$ is a scalar if it transforms under $\mathrm{GC}$ as

$$
\phi(x) \rightarrow \phi^{\prime}(x)=\phi(f(x))
$$

We can write this in a more suggestive way in terms of functional composition:

$$
\phi \rightarrow \phi \circ f
$$

Similarly a vector field $a_{\mu}(x)$ transforms under GC as

$$
a_{\mu}(x) \rightarrow \frac{\partial f^{\alpha}}{\partial x^{\mu}}(x) a_{\alpha}(f(x))
$$

We can write this more compactly by treating $a$ as a form: $\mathbf{a}=a_{\mu}(x) \mathbf{d} x^{\mu}$. Then we just write

$$
\mathbf{a} \rightarrow \mathbf{a} \circ f
$$

where it is understood that $\mathbf{d} x^{\mu} \rightarrow \mathbf{d} f^{\mu}=\partial_{\alpha} f^{\mu} \mathbf{d} x^{\alpha}$. This clearly generalizes to all tensor fields. For example, the transformation properties of the metric $g_{\mu \nu}(x)$ are encoded in $\mathbf{g}=$ $g_{\mu \nu}(x) d x^{\mu} \otimes d x^{\nu}$ as $\mathbf{g} \rightarrow \mathbf{g} \circ f$. Written in this way, coordinate transformations look very 


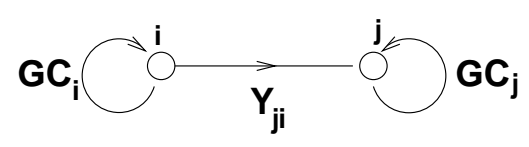

Figure 1: Sites $i$ and $j$ with corresponding $\mathrm{GC}_{i} \times \mathrm{GC}_{j}$ symmetries, connected by a link $Y_{j i}$. Under $\mathrm{GC}_{i} \times \mathrm{GC}_{j}, Y_{j i} \rightarrow f_{j}^{-1} \circ Y_{j i} \circ f_{i}$.

similar to gauge transformations on fields in a gauge theory, e.g. $\phi^{\dagger} \rightarrow \phi^{\dagger} g$, except we have composition in the place of group multiplication.

Now, suppose we have two different sites $i, j$, with two different general coordinate invariances $\mathrm{GC}_{i, j}$. We would like to be able to compare fields on the two sites, which are charged under the different groups. In the gauge theory case, this is accomplished by introducing a link field $U_{j i}$ which transforms under gauge transformations as $U_{j i} \rightarrow g_{j}^{-1} U_{j i} g_{i}$. The object $\left(\phi_{j}^{\dagger} U_{j i}\right)$ transforms only under $g_{i}$ as $\left(\phi_{j}^{\dagger} U_{j i}\right) \rightarrow\left(\phi_{j}^{\dagger} U_{j i}\right) g_{i}$. Similarly, in our case, we introduce a link field $Y_{j i}$ which transforms under $\mathrm{GC}_{i} \times \mathrm{GC}_{j}$ as

$$
Y_{j i} \rightarrow f_{j}^{-1} \circ Y_{j i} \circ f_{i}
$$

Explicitly, the link field $Y_{j i}^{\mu}\left(x_{i}\right)$ is a mapping from the site $i$ to the site $j$ (see figure 1). It associates a point on the site $i$ with coordinate $x_{i}^{\mu}$ with a point on the site $j$ with coordinate $Y^{\mu}\left(x_{i}\right)$; and under $\mathrm{GC}_{i} \times \mathrm{GC}_{j}$, we have

$$
Y_{j i}^{\mu}\left(x_{i}\right) \rightarrow\left(f_{j}^{-1}\right)^{\mu}\left(Y_{j i}\left(f_{i}\left(x_{i}\right)\right)\right)
$$

$Y_{j i}$ is a pullback map from site $j$ to site $i$.

It is now clear how we can compare fields on different sites using the link fields. Suppose we have a field $\psi_{i}$ on site $i$ and a field $\psi_{j}$ on site $j$ which transform under $\mathrm{GC}_{i}$ and $\mathrm{GC}_{j}$ respectively as:

$$
\psi_{i} \rightarrow \psi_{i} \circ f_{i}, \quad \psi_{j} \rightarrow \psi_{j} \circ f_{j},
$$

We can construct an object $\Psi$ out of $\psi_{j}$ which transforms under $\mathrm{GC}_{i}$ by forming

$$
\Psi=\psi_{j} \circ Y_{j i} \Rightarrow \Psi \rightarrow \Psi \circ f_{i}
$$

Let us see how this works explicitly for various tensor fields. Starting with a scalar $\phi_{j}\left(x_{j}\right)$ under $\mathrm{GC}_{j}$, we can form the field

$$
\Phi\left(x_{i}\right)=\phi_{j}\left(Y_{j i}\left(x_{i}\right)\right)
$$

which transforms as a scalar under $\mathrm{GC}_{i}$. Similarly, out of a vector $a_{j \mu}\left(x_{j}\right)$ or a metric $g_{j \mu \nu}\left(x_{j}\right)$, we can form the objects

$$
A_{\mu}\left(x_{i}\right)=\frac{\partial Y^{\alpha}}{\partial x_{i}^{\mu}}\left(x_{i}\right) a_{j \alpha}\left(Y_{j i}\left(x_{i}\right)\right), \quad G_{\mu \nu}\left(x_{i}\right)=\frac{\partial Y^{\alpha}}{\partial x_{i}^{\mu}}\left(x_{i}\right) \frac{\partial Y^{\beta}}{\partial x_{i}^{\nu}}\left(x_{i}\right) g_{j \alpha \beta}\left(Y_{j i}\left(x_{i}\right)\right)
$$

which transform respectively as a vector and metric under $\mathrm{GC}_{i}$. 
Note also that these expressions have the structure of induced tensors familiar from brane dynamics. We can view the site $i$ as a spacetime filling "brane" embedded in the world $j$. $Y_{j i}\left(x_{i}\right)$ is the location of a given point $x_{i}$ on the brane, in the coordinates of the spacetime in which the brane is embedded.

The important point is that we can low write down a Lagrangian like:

$$
\mathcal{L}=\sqrt{g_{i}} g_{i}^{\mu \nu}\left(g_{i \mu \nu}-G_{\mu \nu}\right) g_{i}^{\rho \sigma}\left(g_{i \rho \sigma}-G_{\rho \sigma}\right)
$$

which is invariant under both $\mathrm{GC}_{i}$ and $\mathrm{GC}_{j}$. We can often fix a "unitary gauge" where $Y=\mathrm{id}$ and this Lagrangian becomes:

$$
\mathcal{L}=\sqrt{g_{i}} g_{i}^{\mu \nu}\left(g_{i \mu \nu}-g_{j \mu \nu}\right) g_{i}^{\rho \sigma}\left(g_{i \rho \sigma}-g_{j \rho \sigma}\right)
$$

which is exactly what we need to create mass terms for gravitons. (Lagrangians of the form (3.12) have been considered in [25,26].) Introducing the $Y^{\prime} s$ has allowed us to introduce these mass terms in a way that is formally fully generally covariant. Just as in the analysis of the massive gauge boson, introducing the $Y^{\prime} s$ with this "fake" general covariance will nevertheless be extremely useful in understanding the structure of the theory.

\subsection{Explicit Goldstone boson expansion}

While the above construction is somewhat abstract, it is straightforward to expand $Y$ and $G$ in terms of pions and see how the two general coordinate invariances are realized explicitly. The unitary gauge has $Y=\mathrm{id}$, that is $Y_{j i}^{\mu}\left(x_{i}\right)=x_{i}^{\mu}$. The transformations that leave $Y_{j i}=\mathrm{id}$ are the diagonal subgroup of $\mathrm{GC}_{i} \times \mathrm{GC}_{j}$, where $f_{i}=f_{j}$. Even in situations with many $Y$ fields where we cannot gauge fix all the $Y^{\prime}$ 's to the identity, it is useful to expand the $Y^{\prime}$ around a common $x$ as this corresponds to the small fluctuations around a common background space. We therefore expand $Y$ as

$$
Y^{\alpha}(x)=x^{\alpha}+\pi^{\alpha}(x)
$$

where here and in what follows we have dropped the $i j$ indices on the $Y$ and the $i$ index on $x$ to avoid notational clutter.

Then, the object $G_{\mu \nu}$, from $(3.10)$, can be expanded as:

$$
\begin{aligned}
G_{\mu \nu}= & \frac{\partial Y^{\alpha}(x)}{\partial x^{\mu}} \frac{\partial Y^{\beta}(x)}{\partial x^{\nu}} g_{\alpha \beta}^{j}(Y(x))=\frac{\partial\left(x^{\alpha}+\pi^{\alpha}\right)}{\partial x^{\mu}} \frac{\partial\left(x^{\beta}+\pi^{\beta}\right)}{\partial x^{\nu}} g_{\alpha \beta}^{j}(x+\pi) \\
= & \left(\delta_{\mu}^{\alpha}+\pi_{, \mu}^{\alpha}\right)\left(\delta_{\nu}^{\beta}+\pi_{, \nu}^{\beta}\right)\left(g_{\alpha \beta}^{j}+\pi^{\mu} g_{\alpha \beta, \mu}^{j}+\frac{1}{2} \pi^{\mu} \pi^{\nu} g_{\alpha \beta, \mu, \nu}^{j}+\cdots\right) \\
= & g_{\mu \nu}^{j}+\pi^{\lambda} g_{\mu \nu, \lambda}^{j}+\pi_{, \mu}^{\alpha} g_{\alpha \nu}^{j}+\pi_{, \nu}^{\alpha} g_{\alpha \mu}^{j}+\frac{1}{2} \pi^{\alpha} \pi^{\beta} g_{\mu \nu, \alpha, \beta}^{j} \\
& +\pi_{, \mu}^{\alpha} \pi_{, \nu}^{\beta} g_{\alpha \beta}^{j}+\pi_{, \mu}^{\alpha} \pi^{\beta} g_{\alpha \nu, \beta}^{j}+\pi_{, \nu}^{\alpha} \pi^{\beta} g_{\mu \alpha, \beta}^{j}+\cdots
\end{aligned}
$$

Now we will look at the transformation properties of $g, G, Y$ and $\pi$, under infinitesimal general co-ordinate transformations generated by $f_{i}(x)=x+\varepsilon_{i}(x)$ and $f_{j}(x)=x+\varepsilon_{j}(x)$. 
The metrics on the sites transform as:

$$
\begin{aligned}
& \delta g_{\mu \nu}^{i}=\varepsilon_{i}^{\lambda} g_{\mu \nu, \lambda}^{i}+\varepsilon_{i, \mu}^{\lambda} g_{\lambda \nu}^{i}+\varepsilon_{i, \nu}^{\lambda} g_{\mu \lambda}^{i} \\
& \delta g_{\mu \nu}^{j}=\varepsilon_{j}^{\lambda} g_{\mu \nu, \lambda}^{j}+\varepsilon_{j, \mu}^{\lambda} g_{\lambda \nu}^{j}+\varepsilon_{j, \nu}^{\lambda} g_{\mu \lambda}^{j} \\
& \delta \sqrt{g_{i}}=\varepsilon_{i, \lambda}^{\lambda} \sqrt{g_{i}}+\varepsilon_{i}^{\lambda}\left(\sqrt{g_{i}}\right)_{, \lambda}, \quad \delta \sqrt{g_{j}}=\varepsilon_{j, \lambda}^{\lambda} \sqrt{g_{j}}+\varepsilon_{j}^{\lambda}\left(\sqrt{g_{j}}\right)_{, \lambda}
\end{aligned}
$$

Thus a Lagrangian like

$$
\mathcal{L}=\sqrt{g_{i}} R\left(g_{i}\right)+\sqrt{g_{j}} R\left(g_{j}\right)
$$

trivially has two general coordinate invariances. If we make the replacements (3.15)-(3.17), with independent $\varepsilon_{i}$ and $\varepsilon_{j}$ this Lagrangian is unchanged. Note, however, that the "hopping" Lagrangian in (3.12) is only invariant under the diagonal subgroup for which $\varepsilon_{i}=\varepsilon_{j}$.

Now, the transformation laws of the pions come from transformation of the link $Y$. First, under $\mathrm{GC}_{i}$ :

$$
\begin{aligned}
Y(x) \rightarrow Y\left(x^{\prime}\right) & =x+\varepsilon_{i}+\pi\left(x+\varepsilon_{i}\right) \equiv x+\pi+\delta \pi \\
\Rightarrow \delta \pi^{\mu} & =\varepsilon_{i}^{\mu}+\varepsilon_{i}^{\alpha} \pi_{, \alpha}^{\mu}
\end{aligned}
$$

Under $\mathrm{GC}_{j}$

$$
\begin{gathered}
Y \rightarrow Y-\varepsilon_{j}(Y)=x+\pi-\varepsilon_{j}(x+\pi) \equiv x+\pi+\delta \pi \\
\Rightarrow \delta \pi^{\mu}=-\varepsilon_{j}^{\mu}(x+\pi)=-\varepsilon_{j}^{\mu}-\pi^{\alpha} \varepsilon_{j, \alpha}^{\mu}-\frac{1}{2} \pi^{\alpha} \pi^{\beta} \varepsilon_{j, \alpha, \beta}^{\mu}+\cdots
\end{gathered}
$$

So the pions transform under the two transformations as:

$$
\delta \pi^{\mu}=\varepsilon_{i}^{\mu}+\varepsilon_{i}^{\beta} \pi_{, \beta}^{\mu}-\varepsilon_{j}^{\mu}-\pi^{\beta} \varepsilon_{j, \beta}^{\mu}-\frac{1}{2} \pi^{\alpha} \pi^{\beta} \varepsilon_{j, \alpha, \beta}^{\mu}-\cdots
$$

Note that in the global symmetry limit, where the $\varepsilon$ 's are constant, we have

$$
\pi^{\mu} \rightarrow \pi^{\mu}+\varepsilon_{i}^{\nu} \pi_{, \nu}^{\mu}+\varepsilon_{i}-\varepsilon_{j}=\pi\left(x+\varepsilon_{i}\right)+\varepsilon_{i}-\varepsilon_{j}
$$

This is just a translation in $x_{i}$ by $\varepsilon_{i}$, together with a shift symmetry. Note that in this global limit the symmetry is Abelian. The shift symmetry is the analog of the shift symmetry acting on scalar Goldstone bosons that keeps them exactly massless.

The transformations of the the pions (3.21) are non-linear and messy. But $G_{\mu \nu}$ has simple transformation properties which come from the simple transformations of $Y$. By plugging (3.16) and (3.21) into (3.14) we find that:

$$
\delta G_{\mu \nu}=\varepsilon_{i}^{\lambda} G_{\mu \nu, \lambda}+\varepsilon_{i, \mu}^{\lambda} G_{\lambda \nu}+\varepsilon_{i, \nu}^{\lambda} G_{\mu \lambda}
$$

$G_{\mu \nu}$ transforms like a tensor under $\mathrm{GC}_{i}$ and is invariant under $\mathrm{GC}_{j}$. This is exactly what we wanted. We can now see that the Lagrangian (3.11) possesses two separate general coordinate symmetries: if we make the transformations (3.15) and (3.23) with independent $\varepsilon_{i}$ and $\varepsilon_{j}$ the Lagrangian is unchanged. 


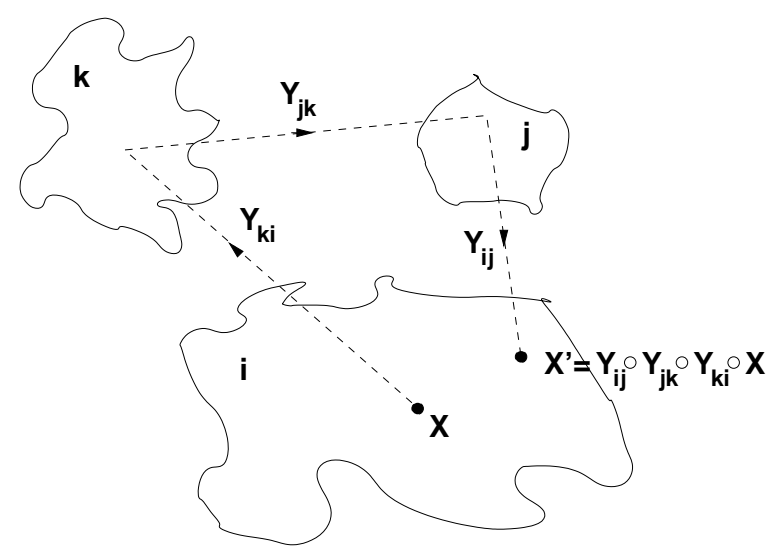

Figure 2: Making a plaquette from $Y_{j i} \circ Y_{i k} \circ Y_{k j}$.

We can also see that is easy to make $G_{\mu \nu}$ invariant under both $\mathrm{GC}_{i}$ and $\mathrm{GC}_{j}$. We just redefine the non-linear transformation laws of $\pi$ to not depend on $\varepsilon_{i}$ by setting $\varepsilon_{i}=0$ in (3.21). Then, without changing the expansion of $G$ in terms of $\pi$ and $g_{j}$, (3.14), $G$ will be invariant for any $\varepsilon_{i}$ and $\varepsilon_{j}$. This is a handy way to add general coordinate invariance to any Lagrangian, even one without a symmetry under the diagonal subgroup. Again, this symmetry is a complete fake, but it can still be useful.

\subsection{Plaquettes}

In the gauge theory case, higher-dimensional theory spaces can be constructed with the appropriate mesh of sites and links. In the case of more than one extra dimension, not all of the link fields can be gauged away. The classical spectrum includes the usual KK tower of spin one particles, but also a large number of massless scalars corresponding to the uneaten link fields. It is possible to give mass to these extra massless fields through the addition of "plaquette" interactions. For example, going in a closed circle of links from $i$ to $j$ to $k$ back to $i$, we can form the plaquette $\left(U_{i j} U_{j k} U_{k i}\right)$, which is conjugated under the action of $g_{i}$. Taking the trace the yields an invariant potential that can be added to the Lagrangian.

We can construct plaquettes in the gravitational case as well. Suppose we have three sites $i, j, k$ with links $Y_{i j}, Y_{j k}, Y_{k i}$. We can form the functional product

$$
\Psi=Y_{i j} \circ Y_{j k} \circ Y_{k i}
$$

which transforms as

$$
\Psi \rightarrow f_{i}^{-1} \circ \Psi \circ f_{i}
$$

How can we build an invariant out of this quantity that can be added to the action? What is the analogue of the trace in the gauge theory case? Note that $\Psi$ maps a point $X$ on site $i$ to a different point $X^{\prime}=\Psi(X)$ (see figure 2). Then the geodesic distance between $X$ and $X^{\prime}$ is coordinate independent and transforms like a scalar under $\mathrm{GC}_{i}$. The simplest 
invariant we can build which is analytic in the fields is then:

$$
\int d^{4} x \sqrt{g_{i}} l^{2}\left(x, \Psi(x) ; g_{i}\right)
$$

where $l[x, y ; g]=\int_{x}^{y} d s$ is the geodesic distance between the points $x, y$ with the metric $g$. (We are imagining that all the $\pi$ 's are perturbatively close to zero, so that there is no ambiguity in which is the shortest geodesic between $x$ and $\Psi(x)$.) This is the analog of the simplest single trace plaquette operator in the gauge theory. Expanding around flat space, this operator gives a mass to the uneaten combination of Goldstones $\pi_{i j}^{\alpha}+\pi_{j k}^{\alpha}+\pi_{k i}^{\alpha}$ and provides additional non-linear interactions needed to preserve the general covariances. This is all in complete analogy with the gauge theory case.

\section{Massive gravitons}

We will now show how the effective field theory formalism of the previous section makes studying a massive graviton embarrassingly easy. These fields have been sporadically studied for many years, and are known to have several peculiar properties. Almost all of the studies have been based on deforming GR by the addition of the (already somewhat peculiar) FierzPauli mass term, $\left(h_{\mu \mu}\right)^{2}-h_{\mu \nu} h_{\mu \nu}$, where $h_{\mu \nu}=g_{\mu \nu}-\eta_{\mu \nu}$ is the metric linearized around flat space. This specific linear combination is needed for a unitary propagator $[10,11]$. Then there is the famous van Dam-Veltman-Zakharov discontinuity $[12,13]$ in the graviton propagator as $m_{g} \rightarrow 0$, which seems to indicate that the an arbitrarily small mass graviton yields different predictions than Einstein's theory. Recently, it has been observed [14-18, 20]. that this discontinuity disappears in Anti-de-Sitter space and in de Sitter space, though there is a ghost instead in the de Sitter theory. Finally, there is the observation of Vainshtein [19], that the discontinuity may not be relevant for physical sources, because the linearized approximation to gravity outside a source of mass $M$ breaks down at a much larger distance than the gravitational radius $R_{g}=l_{P l}^{2} M$; at a distance $\left(m_{g}^{-4} R_{g}\right)^{1 / 5}$.

With our Goldstone boson description, we will understand these peculiarities trivially, and see that they are all associated with a single underlying cause. The scalar longitudinal component of the graviton becomes strongly coupled at a far lower energy scale than we may have expected by analogy with the familiar gauge theory case. For a massive spin one field, the cutoff is $\sim m_{A} / g$. This would translate to a cutoff $\sim \sqrt{m_{g} M_{\mathrm{Pl}}}$ in the gravity case. However, we will find that while there is a sensible effective theory for interacting massive gravitons, the cutoff is parametrically far lower than this. Beginning with the Fierz-Pauli Lagrangian,

it is $\sim\left(m_{g}^{4} M_{\mathrm{Pl}}\right)^{1 / 5}$, while a slightly more clever starting point can push the cutoff higher to $\sim\left(m_{g}^{2} M_{\mathrm{Pl}}\right)^{1 / 3}$.

\subsection{Two site model}

Following the gauge example, it is straightforward to isolate a single massive graviton in an arbitrary background from a two site model. We start with an action is of the form

$$
S=S_{\text {grav }}+S_{\text {mass }}
$$


where

$$
S_{\text {grav }}=\int d^{4} x \sqrt{-g}\left(M_{\mathrm{Pl}}^{2} R[g]+\cdots\right)+\int d^{4} x_{0} \sqrt{-g_{0}}\left(-\Lambda_{0}+M_{0}^{2} R\left[g_{0}\right]+\cdots\right)
$$

represents the action for the gravitons on the sites. For simplicity we have not put in a cosmological constant term on the first site. And

$$
S_{\text {mass }}=\int d^{4} x \sqrt{-g} g^{\mu \nu} g^{\alpha \beta}\left(a H_{\mu \nu} H_{\alpha \beta}+b H_{\mu \alpha} H_{\nu \beta}\right)+\cdots
$$

denotes the "hopping" action that will give one combination of gravitons a mass. Here,

$$
H_{\mu \nu}(x) \equiv g_{\mu \nu}(x)-\partial_{\mu} Y^{\alpha}(x) \partial_{\nu} Y^{\beta}(x) g_{0 \alpha \beta}(Y(x))
$$

We can go to a unitary gauge where $Y=\mathrm{id}$ and there is one manifest general coordinate invariance under which both $g$ and $g_{0}$ transform as tensors. The spectrum contains one massless graviton and one massive graviton. In the limit where we send $M_{0} \rightarrow \infty$, this massless graviton is all $g_{0}$ and becomes non-dynamical, and so we are left with a theory of a single massive graviton described by $g$, in a non-dynamical background geometry $g_{0}$. (W. Siegel has informed us that this way of introducing general coordinate invariance for a massive graviton was considered in [28]. For other early work see [29].) The Goldstone formulation will be invaluable in elucidating the interactions of the longitudinal components of the massive gravitons and determining the structure of the effective field theory.

\subsection{Linearized analysis}

Let us begin by analyzing our action to quadratic order in the fields. To wit, $H_{\mu \nu}$ is expanded as in (3.14) with $g_{j}=g_{0}$ :

$$
H_{\mu \nu}=h_{\mu \nu}+g_{0 \mu \alpha} \nabla_{\nu}^{0} \pi^{\alpha}+g_{0 \nu \alpha} \nabla_{\mu}^{0} \pi^{\alpha}+\cdots
$$

where

$$
h_{\mu \nu} \equiv g_{\mu \nu}-g_{0 \mu \nu}
$$

and $\nabla_{\mu}^{0}$ is the covariant derivative with the background metric $g_{0}$.

Our Goldstones are a vector field, which has 3 polarizations. These are eaten by the massless graviton, which has 2 polarizations, to produce a massive graviton with a total of 5 physical polarizations. We can decompose the $\pi^{\alpha}$ into the transverse spin one and scalar mode by expressing

$$
\pi^{\alpha}(x)=g_{0}^{\alpha \beta}\left(A_{\beta}+\partial_{\beta} \phi\right)
$$

This allows us to introduce a fake $U(1)$ gauge symmetry under which

$$
A_{\beta} \rightarrow A_{\beta}+\partial_{\beta} \Lambda, \phi \rightarrow \phi-\Lambda
$$

The graviton mass term will turn into the kinetic term for these Goldstones. Of course, as usual the new fake general covariance and $U(1)$ symmetries must be gauge fixed by the 
addition of suitable gauge fixing terms for $h_{\mu \nu}$ and $A_{\alpha}$, for instance fixing to Feynman-like gauges for both. The precise form of this gauge fixing will not be relevant for our discussion. Note that as defined $A_{\alpha}$ has mass dimension -1 and $\phi$ has mass dimension -2 .

By the usual logic leading to the equivalence theorem for gauge theories, we expect that the physics of $A$ and $\phi$ is that of the vector longitudinal $\left(g^{\mathrm{vL}}\right)$ and scalar longitudinal $\left(g^{\mathrm{sL}}\right)$ polarizations of the massive graviton field, at energies much higher than the mass of the graviton:

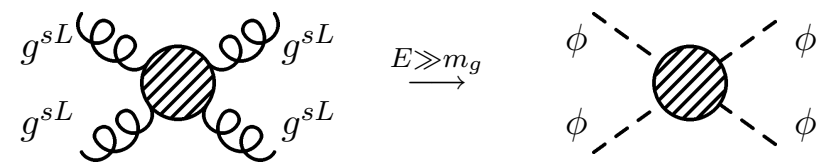

Let us now consider the case where the background $g_{0 \mu \nu}=\eta_{\mu \nu}$ is flat. We can immediately notice a peculiarity that will be at the heart of the difference the gravity and gauge theory cases. In the global symmetry limit where we send $M_{\mathrm{Pl}} \rightarrow \infty$ ( equivalently set $h_{\mu \nu} \rightarrow 0$ ), there is a shift symmetry on the $\pi^{\alpha}$ that means that $\pi^{\alpha}$ only appears with a derivative acting on it. This implies that $\phi$ only ever appears with two derivatives, so $\phi^{2}$ must have four derivatives. Therefore, $\phi$ cannot have a normal kinetic term in this limit. Indeed, for general coefficients $a$ and $b$ in $S_{m}, \phi$ will have a four-derivative kinetic term as

$$
\begin{aligned}
S_{\text {mass }} & \supset \int d^{4} x 4 a \phi_{, \mu, \nu} \phi_{, \mu, \nu}+4 b \square \phi \square \phi \\
& =\int d^{4} x 4(a+b) \square \phi \square \phi
\end{aligned}
$$

where to get to the second line we have integrated by parts. In order to eliminate this pathological kinetic term, which implies ghosts and violations of unitarity, we must choose $a+b=0$. This is precisely the Fierz-Pauli mass term in the unitary gauge,

$$
\mathcal{L}_{F P}=f^{4}\left(h_{\mu \nu} h_{\mu \nu}-h^{2}\right)
$$

corresponding to a graviton mass

$$
m_{g}^{2}=\frac{f^{4}}{M_{\mathrm{Pl}}^{2}}
$$

We will assume throughout that the graviton mass is parametrically much smaller than the Planck scale, $m_{g} \ll M_{P l}$; this is essential for any sensible effective theory.

Note that with the Fierz-Pauli choice $a=-b, \phi$ has no kinetic term in the decoupling limit. On the other hand, $A_{\alpha}$ has a perfectly healthy kinetic term $f^{4}\left(A_{\mu, \nu}-A_{\nu, \mu}\right)^{2}$ in this limit. Now, $\phi$ does acquire a normal two-derivative kinetic term, but only via mixing with the graviton $h_{\mu \nu}$. Indeed, in the expansion of $S_{\text {mass }}$, there is is a mixing term

$$
f^{4}\left(h_{\mu \nu} \phi, \mu, \nu-h \square \phi\right)
$$

It is useful to express this term in a more familiar form. After integrating by parts, this kinetic mixing term is $f^{4} \phi R_{\text {lin }}$ where $R_{\text {lin }}=h_{\mu \nu, \mu, \nu}-\square h$ is the Ricci scalar at linear order in $h$. Thus, at quadratic order in the fields the kinetic Lagrangian is the same as

$$
\sqrt{g} M_{P l}^{2}\left(1+m_{g}^{2} \phi\right) R
$$


We can eliminate the kinetic mixing between $\phi$ and $h$ by by a Weyl rescaling of the metric, which is well-known to generate a kinetic term for $\phi$ with the correct sign. At quadratic order this amounts to is redefining

$$
h_{\mu \nu}=\hat{h}_{\mu \nu}-\eta_{\mu \nu}\left(m_{g}^{2} \phi\right)
$$

and the induced kinetic term for $\phi$ is

$$
M_{P l}^{2} m_{g}^{4}(\partial \phi)^{2}=\frac{f^{8}}{M_{P l}^{2}}(\partial \phi)^{2}
$$

It will be also be convenient to add a gauge-fixing term directly for $\hat{h}$. Then at quadratic order, we have usual kinetic and mass terms for $\hat{h}_{\mu \nu}$, and a kinetic term for $\phi$, with no kinetic mixing between them.

So $\phi$ does acquire a normal kinetic term, but this term disappears in the limit where $f$ is held fixed and $M_{\mathrm{Pl}}$ is sent to infinity. This is in stark contrast with the gauge theory case, where the Goldstone scalar kinetic term is $\sim f^{2}(\partial \pi)^{2}$ and survives as $g \rightarrow 0$.

Now, we certainly do not expect our choice $a=-b$ to be exactly radiatively stable at quantum level, so let us get a better idea of what would happen if $a \neq-b$. The $\phi$ kinetic term would have the structure in momentum space

$$
\frac{f^{8}}{M_{\mathrm{Pl}}^{2}} p^{2} \phi^{2}+(a+b) p^{4} \phi^{2}
$$

and this would lead to ghosts or tachyons at a momentum scale $p^{2} \sim \frac{f^{8}}{(a+b) M_{\mathrm{Pl}}^{2}} \sim m_{g}^{2} \frac{f^{4}}{a+b}$. So we see why we need to have $(a+b) \ll f^{4}$, because otherwise our effective theory would break down right around the mass of the particle $m_{g}$ and would be completely useless. This is the analog of the reason why in gauge theory we do not include a $\frac{1}{g^{2}}(\partial A)^{2}$ kinetic term. As we saw, such a term is generated at quantum level, but with a small enough coefficient so that its harmful effects are deferred to the cutoff. We will see that exactly the same thing happens in our case; with a suitable cutoff, a small $(a+b)$ is generated (along with a whole slew of other terms), but with small enough sizes to allow the effective theory to make sense to energies parametrically above $m_{g}$.

Notice that in order to go to canonical normalization for $\phi$, we define

$$
\phi=\frac{M_{\mathrm{Pl}}}{f^{4}} \phi^{c}=\frac{1}{m_{g}^{2} M_{\mathrm{Pl}}} \phi^{c}
$$

The $M_{\mathrm{Pl}}$ in the numerator implies that the interactions of $\phi$ will become strongly coupled at an energy far beneath $f$, again in contrast with the gauge theory case. On the other hand, the $A_{\alpha}$ kinetic term is proportional to $\sim f^{4}$, and the canonically normalized field is

$$
A_{\alpha}=\frac{1}{f^{2}} A_{\alpha}^{c}=\frac{1}{m_{g} M_{\mathrm{Pl}}} A_{\alpha}^{c}
$$

These conclusions are changed in a general curved background, which introduces another scale into the problem. In flat space, with the choice $a=-b=f^{4}$ there is no kinetic term for 
$\phi$ without mixing through $h$ : the contributions to the $\phi$ kinetic term proportional to $a$ and $b$ cancel exactly after integrating by parts. In a general curved background, we have instead at quadratic order

$$
\begin{aligned}
f^{4} \int d^{4} x \sqrt{-g_{0}} & \left(\nabla_{\mu}^{0} \nabla_{\nu}^{0} \phi \nabla^{0 \mu} \nabla^{0 \nu} \phi-\left(\nabla^{0}\right)^{2} \phi\left(\nabla^{0}\right)^{2} \phi\right) \\
& =f^{4} \int d^{4} x \sqrt{-g_{0}} \phi_{, \mu}\left[\nabla^{0 \mu}, \nabla^{0 \nu}\right] \phi_{, \nu}
\end{aligned}
$$

after integration by parts. Since the commutator of the covariant derivatives is proportional to the Riemann tensor and is non-vanishing in a curved background, there is an induced kinetic term for $\phi$ (and a corresponding mass term for $A_{\alpha}$ ) proportional to the background curvature. For a maximally symmetric space like AdS the $\left[\nabla^{0 \mu}, \nabla^{0 \mu}\right]$ term can be replaced by $\frac{1}{L^{2}} g^{0 \mu \nu}$ in (4.22), where $L$ is AdS radius of curvature. Therefore this contribution to the $\phi$ kinetic term is

$$
\frac{f^{4}}{L^{2}}(\partial \phi)^{2}=\frac{m_{g}^{2}}{L^{2}} M_{\mathrm{Pl}}^{2}(\partial \phi)^{2}
$$

This should be compared with the kinetic term coming from mixing with $h, \sim m_{g}^{4} M_{\mathrm{Pl}}^{2}(\partial \phi)^{2}$. We see that for $1 / L \gg m_{g}$, the new contribution to the kinetic term dominates. It is easy to check that it has the good sign in AdS space and the bad sign leading to ghosts in dS space. Note that for $1 / L \gg m_{g}$, the kinetic term survives as $M_{\mathrm{Pl}}$ is taken to infinity with $f$ fixed, and in this limit the canonically normalized $\phi$ field is

$$
\phi=\frac{L}{f^{2}} \phi^{c}=\frac{1}{m_{g} L M_{\mathrm{Pl}}} \phi^{c}\left(\text { for } \frac{1}{L} \gg m_{g}\right)
$$

\subsection{The vDVZ discontinuity in general backgrounds}

We are now in a position to easily understand the presence/absence of the vDVZ discontinuity in general spacetimes. Let us first work in flat space. The coupling of the graviton $h_{\mu \nu}$ to the energy momentum tensor is

$$
T_{\nu}^{\mu} h_{\mu}^{\nu}=T_{\nu}^{\mu}\left(\hat{h}_{\mu}^{\nu}+m_{g}^{2} \delta_{\mu}^{\nu} \phi\right)=T_{\nu}^{\mu} \hat{h}_{\mu}^{\nu}+\frac{1}{M_{\mathrm{Pl}}} T \phi^{c}
$$

where in the last equality we have gone to canonical normalization for $\phi$. We see that independent of the graviton mass $m_{g}$, there is a coupling of the trace of the energy momentum tensor to $\phi$ with gravitational strength. Thus, for $m_{g} \rightarrow 0$ matter and radiation would couple with different relative strengths than they would if $\phi$ were absent, that is if $m_{g}$ were strictly massless. This is exactly the vDVZ discontinuity, and we have traced its origin to the strongly coupled nature of the scalar $\phi$. Despite the fact that $h_{\mu \nu}$ only has a small admixture $m_{g}^{2} \eta_{\mu \nu} \phi$ of $\phi$ which appears to go to zero as $m_{g} \rightarrow 0$, because the $\phi$ kinetic term vanishes in the same limit, when we go to canonical normalization the scalar couples with gravitational strength independent of $m_{g}$. 
Then we can see immediately that this discontinuity vanishes in an AdS background. There is still a coupling $m_{g}^{2} \phi T$, however in the limit where $1 / L \gg m_{g}$, in going to canonical normalization $\phi=L /\left(m_{g} M_{\mathrm{Pl}}\right) \phi^{c}$ we obtain the coupling

$$
\frac{m_{g} L}{M_{\mathrm{Pl}}} T \phi^{c}
$$

which does vanishes as $m_{g} \rightarrow 0$. There is therefore no new gravitational strength force and no vDVZ discontinuity in this limit.

\subsection{Strong coupling scale and power-counting in the effective theory}

Let us now return to flat space but consider the fully interacting theory. We are in particular interested in the interactions of the longitudinal components of the graviton at energies far above $m_{g}$, which are the interactions of the $\phi$ and $A_{\alpha}$. These are the strongest interactions and signal when the theory breaks down.

In flat space, our expression for $H_{\mu \nu}$ in terms of the Goldstone bosons becomes

$$
H_{\mu \nu}=h_{\mu \nu}+\pi_{\mu, \nu}+\pi_{\nu, \mu}+\pi_{\alpha, \mu} \pi_{\alpha, \nu}
$$

where $\pi_{\mu}=\eta_{\mu \nu} \pi^{\nu}$. Since we are not interested in the usual helicity two polarization, we can set $\hat{h}_{\mu \nu}=0$. Furthermore, since $\hat{h}_{\mu \nu}$ and $h_{\mu \nu}$ only differ by an amount that is $\sim 1 / M_{\mathrm{Pl}} \phi^{c} \eta_{\mu \nu}$, for the amplitudes of interest which will be getting large far beneath $M_{\mathrm{Pl}}$ we can simply set $h_{\mu \nu}=0$ in all interaction terms. Thus, to obtain the interactions for our Goldstones, it suffices to replace everywhere

$$
H_{\mu \nu} \rightarrow \pi_{\mu, \nu}+\pi_{\nu, \mu}+\pi_{\alpha, \mu} \pi_{\alpha, \nu}
$$

The Fierz-Pauli mass term can then be seen to contain cubic and quartic interactions for $\phi$ and $A$. The only interactions that can become anomalously large involve $\phi$, and are schematically

$$
f^{4}\left[\left(\partial^{2} \phi\right)^{3}+\left(\partial^{2} \phi\right)^{4}+\partial^{2} \phi \partial A \partial A\right]
$$

(Note that it is impossible to have a term with a single $\partial A$ and $\partial^{2} \phi$ 's, because by the $U(1)$ gauge invariance (4.8), the $\partial A$ piece would have to involve $F_{\mu \nu}$ which is antisymmetric, and vanishes when contracted with anything made out of $\phi_{, \mu, \nu}$ which is symmetric in $\mu, \nu$.)

Going to canonical normalization, these interactions become

$$
\frac{1}{m_{g}^{4} M_{\mathrm{Pl}}}\left(\partial^{2} \phi^{c}\right)^{3}+\frac{1}{m_{g}^{6} M_{\mathrm{Pl}}^{2}}\left(\partial^{2} \phi^{c}\right)^{4}+\frac{1}{m_{g}^{2} M_{\mathrm{Pl}}} \partial^{2} \phi^{c} \partial A^{c} \partial A^{c}
$$

The cubic scalar interaction is the strongest coupling and becomes large at an energy scale

$$
\Lambda_{5} \sim\left(m_{g}^{4} M_{\mathrm{Pl}}\right)^{1 / 5}
$$

Correspondingly, the amplitude $\mathcal{A}(\phi \phi \rightarrow \phi \phi)$ from $\phi$ exchange grows as $\sim E^{10} / \Lambda_{5}^{10}$ and gets strongly coupled at $\Lambda_{5}$. This means that the scattering amplitude for the scalar longitudinal polarization of the massive graviton gets strongly coupled at the scale $\Lambda_{5}$ : 


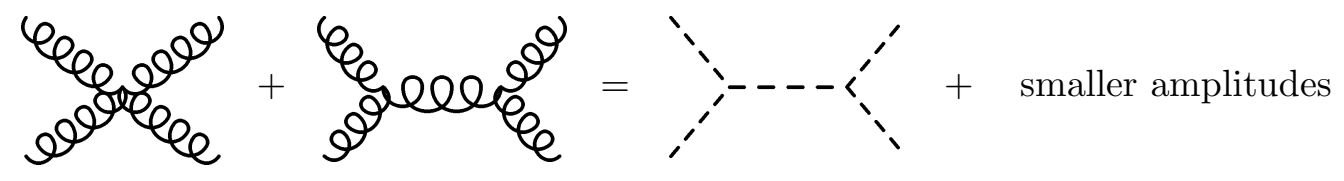

This could have actually been guessed directly in unitary gauge. The polarization vector for the scalar longitudinal polarization of the graviton at high energies is $\epsilon_{\mu \nu} \sim k_{\mu} k_{\nu} / m_{g}^{2} \sim$ $\left(E^{2} / m_{g}^{2}\right)$. Naively, each one of the graviton diagrams in (4.32) grow as

$$
\left(\frac{E^{2}}{m_{g}^{2}}\right)^{4} \times \frac{E^{2}}{M_{\mathrm{Pl}}^{2}} \sim \frac{E^{10}}{\Lambda_{5}^{10}}
$$

In the gauge theory case, there is a cancellation of the leading term between the two diagrams; one may have expected a similar cancellation here. However, without the need to perform this very hairy perturbative massive gravity computation, our Goldstone description tells us that no such cancellation occurs, and the amplitude gets strongly coupled at $\Lambda_{5}$. Perhaps this is not surprising, since our starting point, the Fierz-Pauli Lagrangian, is somewhat arbitrary. That is, there is nothing special about terms quadratic in $h$ given that $h$ is dimensionless. Higher order interactions may help cancel the strongest divergences in 4.32). We will come back to this in section 4.5 .

Let us proceed to determine the structure of the operators generated at quantum level in this effective theory, taken to have a cutoff $\Lambda_{5}$. We must include all operators consistent with the symmetries, suppressed by the cutoff $\Lambda_{5}$. The shift symmetry guarantees that the leading operators are of the form

$$
\frac{\partial^{q}\left(\partial^{2} \phi^{c}\right)^{p}}{\Lambda_{5}^{3 p+q-4}}
$$

In order to find what operators these correspond to in unitary gauge, we can go back to the original normalization for $\phi^{c}=m_{g}^{2} M_{\mathrm{Pl}} \phi$ and recall that $\phi_{, \mu, \nu}$ always comes from an $h_{\mu \nu}$. Thus, in unitary gauge, we have operators of the form

$$
c_{p, q} \partial^{q} h^{p}
$$

where the coefficients $c_{p, q}$ have a natural size

$$
c_{p, q} \sim \Lambda_{5}^{-3 p-q+4} M_{\mathrm{Pl}}^{p} m_{g}^{2 p}=\left(m_{g}^{16-4 q-2 p} M_{\mathrm{Pl}}^{2 p-q+4}\right)^{1 / 5}
$$

Note that, for example, the term with $p=2, q=0$ is a general mass term for $h$, not necessarily of the Fierz-Pauli form. However, its coefficient, $c_{2,0}=\left(m_{g}^{12 / 5} M_{\mathrm{Pl}}^{8 / 5}\right)$, is parametrically much smaller than the Fierz-Pauli coefficient $f^{4}=m_{g}^{2} M_{\mathrm{Pl}}^{2}$ (4.12), and the unitarity violation/tachyons are postponed to energies above the cutoff $\Lambda_{5}$.

We can summarize by saying that there is a natural effective theory with an action

$$
\int d^{4} x \sqrt{-g}\left(M_{\mathrm{Pl}}^{2} R+\cdots\right)+m_{g}^{2} M_{\mathrm{Pl}}^{2}\left(h_{\mu \nu}^{2}-h^{2}\right)+\sum_{p, q} c_{p, q} \partial^{q} h^{p}
$$


with a cutoff $\Lambda_{5}=\left(m_{g}^{4} M_{\mathrm{Pl}}\right)^{1 / 5}$. The "pollution" from all the higher order terms do not give rise to any pathologies until above $\Lambda_{5}$. Needless to say, it would have been hard to guess the structure of this effective theory directly from a unitary gauge analysis.

\subsection{Adding interactions to raise the cutoff}

We can easily find another natural effective theory where the cutoff is parametrically higher than $\Lambda_{5}$. By adding higher-order terms of the form $f^{4}\left(h^{3}+h^{4}+\cdots\right)$, we can remove all the $\phi$ self-couplings from the action. There is no unique procedure, but one way is as follows. Since $H_{\mu \nu} \sim \phi_{, \mu, \nu}+O\left(\left(\partial^{2} \phi\right)^{2}\right)$, at any order we can cancel all terms of the form $\left(\partial^{2} \phi\right)^{n}$ by appropriately choosing the coefficient of $H^{n}$ terms. These in turn only generate higher order $\phi$ self-interactions, which are canceled at the next step. Having eliminated all the $\phi$ self-interactions with terms of the form $f^{4} H^{n}$, we are left with interactions of the form

$$
f^{4}(\partial A)^{p}\left(\partial^{2} \phi\right)^{q}=\frac{1}{m_{g}^{p+2 q-2} M_{\mathrm{Pl}}^{p+q-2}}\left(\partial A^{c}\right)^{p}\left(\partial^{2} \phi^{c}\right)^{q}
$$

for $p>1$. These become strongly coupled at a scale

$$
\left(m_{g}^{p+2 q-2} M_{\mathrm{Pl}}^{p+q-2}\right)^{\frac{1}{3 q+2 p-4}}
$$

It is easy to see that the lowest this scale can ever be is $\Lambda_{3}=\left(m_{g}^{2} M_{\mathrm{Pl}}\right)^{1 / 3}$, which is achieved for $p=2, q=1$, and also asymptotically as $q \rightarrow \infty$. Therefore the cutoff of this effective theory is $\Lambda_{3}$. Note that with this choice, the leading contribution to the $\phi \phi \rightarrow \phi \phi$ amplitude is absent, which means that there is a partial cancellation between the two unitary gauge diagrams on the left side of (4.32). The largest amplitude is that of $A A$ scattering through $\phi$ exchange, where the amplitude again grows as naively expected in unitary gauge $\sim E^{6} /\left(m_{g}^{4} M_{\mathrm{Pl}}^{2}\right)$, becoming strongly coupled at $\sim \Lambda_{3}$. It is easy to find that the natural size for operators of the form $c_{p, q} \partial^{q} h^{p}$ in unitary gauge is now

$$
c_{p, q} \sim \Lambda_{3}^{4-q}=\left(m_{g}^{2} M_{\mathrm{Pl}}\right)^{\frac{4-q}{5}}
$$

Again, the choice of coefficients needed to eliminate all the $\phi$ self-interactions is technically

natural; since $\Lambda_{3} \ll f=\sqrt{m_{g} M_{\mathrm{Pl}}}$, the pollution from the operators not of the special form is small and pathologies are postponed to the cutoff.

This makes it tempting to try to push the cutoff higher by adding other interactions. We will address this question in detail elsewhere [31].

\subsection{Breakdown of the effective theory around heavy sources}

We have seen that our effective field theory breaks down at high energy scales $\Lambda \sim \Lambda_{5}$ or $\Lambda_{3}$, and there are infinitely many higher dimension operators suppressed by $\Lambda$ that encode our ignorance of the short-distance UV completion of these theories. In high-energy scattering experiments, these effects only become important near the cutoff scale. But quite generally, effective theories can also break down at large distance scales in the presence of large 
background fields that make the higher dimension operators important. For instance, the Euler-Heisenberg Lagrangian which describes electrodynamics at energies beneath the electron mass contains higher-dimension operators of the form $\sim F^{4} / m^{4}, F^{6} / m^{8} \cdots$. The effective theory certainly breaks down at short distances of order $m^{-1}$, but it can also break down in backgrounds with spatially homogeneous but large electric fields, where $F / m^{2}$ becomes $\sim 1$. Schwinger pair production becomes important and the structure of the short-distance physics becomes relevant even at large distances. Similarly, in our case there are higher-dimension operators which become important when $\partial^{2} \phi^{c} / \Lambda^{3}$ becomes $\sim 1$, and this quantity can indeed become large in the presence of heavy sources with masses $M$ much larger than $M_{P l}$.

Consider for instance the potential field for $\phi^{c}$. Since $\phi^{c}$ couples with gravitational strength, it will affect the motion of test particles around a heavy source. The potential set up for $\phi^{c}$ by a source of mass $M$ can be diagrammatically represented as

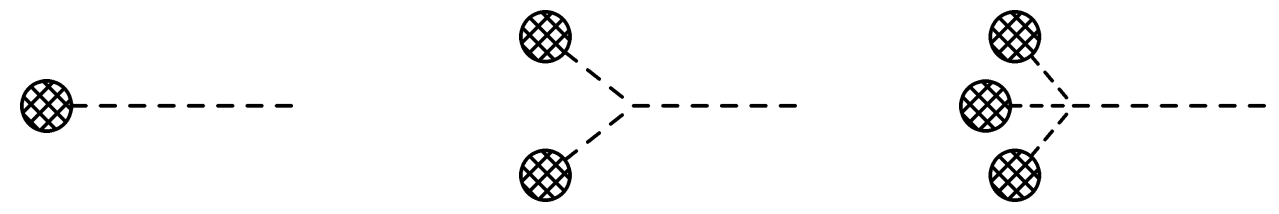

Here the blobs denote the heavy external source. The first diagram represents the potential set up for $\phi^{c}$ at linearized level, which will be a good approximation at sufficiently large distances from the source. The coupling to the source is proportional to $M / M_{\mathrm{Pl}}$ so

$$
\phi^{c(1)} \sim \frac{M}{M_{\mathrm{Pl}}} \frac{1}{r}
$$

The remaining diagrams can be easily estimated. Each vertex gives us a factor of $\left(M / M_{P l}\right)$, while from $n$ point vertices of the form $\partial^{q}\left(\partial^{2} \phi_{c}\right)^{n} / \Lambda^{3 n+q-4}$ there is a factor of $1 / \Lambda^{3 n+q-4}$. The remaining $1 / r$ factors are fixed by dimensional analysis, and we find the contribution to $\phi_{c}$ is

$$
\phi_{c}^{(n, q)} \sim\left(\frac{M}{M_{P l}}\right)^{n-1} \frac{1}{\Lambda^{3 n+q-4}} \frac{1}{r^{3 n+q-3}}
$$

The distance $r_{n}$ at which the $n$ 'th order contribution to $\phi_{c}$ becomes comparable to the lowest order contribution is then

$$
r_{n, q} \sim \Lambda^{-1}\left(\frac{M}{M_{P l}}\right)^{\frac{n-2}{3 n+q-4}}
$$

Note interestingly that this distance increases with $n$, and asymptotes to

$$
r_{*} \sim \Lambda^{-1}\left(\frac{M}{M_{P l}}\right)^{1 / 3}
$$

This distance is precisely where $\partial^{2} \phi^{c(1)} / \Lambda^{3}$ becomes $\sim 1$. In the action, terms with the minimal number of derivatives $q=0$, but with any $n$, all become important at the distance $r_{*}$.

This tells us two things. First, the effective theory around heavy sources breaks down at parametrically much larger distances than the short distance cutoff $\Lambda^{-1}$, by a factor of 
$\left(M / M_{P l}\right)^{1 / 3}$, since infinitely many higher dimension operators become important at this scale. Second, there is no range of distances for which the linear approximation breaks down but non-linear effects can be reliably computed in the effective theory, i.e. for which only a finite number of the higher dimension operators become important. This is because it is the highest dimension operators that contribute to the onset of non-linear effects at large distances. We therefore directly transition from the linear regime to one where the effective theory breaks down. This is in contrast with non-linear effects in Einstein Gravity. Here, at a distance of order the gravitational radius, the linearized approximation breaks down, and all the operators with two derivatives and any number of $h$ 's become important. However, general covariance dictates that all these higher dimension operators are packaged together into the Ricci scalar $R$, and therefore all their coefficients are known. We can therefore trust the nonlinear gravity solution to much smaller distances, and the effective theory only breaks down when the curvatures become Planckian. In our case, we don't have any symmetry principle analgous to general covariance to determine the coefficients of all operators with $q=0$ for any $n$, and therefore without a UV completion we are unable to compute non-linear effects around heavy sources consistently within the effective theory.

It is interesting to compare our conclusions here with the observations of Vainshtein [19] that foreshadowed some of these results. Working with the Fierz-Pauli theory, purely at the classical level, Vainshtein found that the linear approximation for gravity breaks down at a macroscopic distance $r_{V} \sim\left(G_{N} M m_{g}^{-4}\right)^{1 / 5}$ from the source. He further argued that the full non-linear solution would have a continuous behavior as $m_{g}$ is taken to zero. We can understand the origin of the Vainshtein radius trivially. Recall that just with the Fierz-Pauli term, the strongest interaction was the triple-scalar interaction suppressed by $\Lambda_{5}$. Therefore the contribution $n=3, q=0$ in our analysis above is the largest one, and the corresponding radius $r_{3,0}$, where this non-linear correction becomes comparable to the lowest-order term, is precisely $r_{V}$. However, from the point of view of the effective theory, there is no reason to only keep the Fierz-Pauli terms, and in fact we must include all other operators with their natural sizes. Doing this we have found even larger non-linear effects. We have seen that the entire effective theory breaks down at the distance $r_{*}$, so that it is impossible to make any reliable predictions for gravitational strength forces at distances smaller than $r_{*}$ without specifying the UV completion of the theory. And we certainly do not have any reason to expect a smooth limit as $m_{g}$ is taken to zero, since in this limit the distance scale at which the effective theory breaks down goes to infinity.

If we wish to consider the possibility that our four-dimensional graviton has a small mass, then its Compton wavelength should be on order of the size of the universe: $m_{g} \sim 10^{28} \mathrm{~cm}^{-1}$. Then, we find that $\Lambda_{5}^{-1} \sim 10^{13} \mathrm{~cm}$. Even if we modify the theory to raise the cutoff to $\Lambda_{3}$, we would only have $\Lambda_{3}^{-1} \sim 10^{7} \mathrm{~cm}$. The effective theory breaks down at even larger distances around heavy sources. Both of these scales are far larger than $\sim 1 \mathrm{~mm}$, where gravitational effects have been measured. Therefore our effective theory for a massive $4 \mathrm{D}$ graviton breaks down at distances larger than the scale we have measured gravity, and we cannot say in any controlled way that this theory is consistent with experiment. In order to avoid conflict with 
experiment, the short distance cutoff must at least be pushed to $\sim \mathrm{mm}$, which is around $\sqrt{m_{g} M_{P l}}$. We will discuss this possibility in more detail in [31].

\section{Summary, Discussion and Outlook}

We have shown how to understand massive gravitons within the language of effective field theory. We are now able to write down interacting gravitational Lagrangians in a theory space with multiple copies of general coordinate invariance. The key is to introduce link fields which transform non-linearly under various transformations. In unitary gauge, these links are eaten to make the gravitons massive. Our generally covariant formalism allows us to study the largest interaction in the theory, involving the longitudinal components of the massive gravitons, in a simple way.

As an illustration, we have applied this formalism to study a single graviton of mass $m_{g}$. We find that there is a consistent effective theory with a cutoff $\Lambda$ which can be taken

parametrically higher than $m_{g}$. It can be taken as high as $\Lambda \sim\left(M_{\mathrm{Pl}} m_{g}^{4}\right)^{\frac{1}{5}}$ for the simplest case based on the Fierz-Pauli theory, and as high as $\Lambda \sim\left(M_{\mathrm{Pl}} m_{g}^{2}\right)^{\frac{1}{3}}$ if we add additional terms beyond the Fierz-Pauli structure. We have understood a number of strange features of massive gravitons in a transparent way, and seen that they are all consequences of the peculiar behavior of the scalar longitudinal component of the graviton, $\phi$. That the mass term must have Fierz-Pauli form to guarantee unitarity follows immediately from eliminating the pathological large four-derivative kinetic term for $\phi$. Having done this, around flat space $\phi$ only acquires a kinetic term by mixing with $h_{\mu \nu}$. This is the origin of the vDVZ discontinuity. However, around curved backgrounds, such as AdS space, $\phi$ does pick up a normal kinetic term proportional to the background curvature even without mixing, and therefore the vDVZ discontinuity is absent. We have shown how to include all terms beyond the Fierz-Pauli Lagrangian with their natural sizes in the effective theory, and in particular observed that the Fierz-Pauli form of the mass term is radiatively stable. We also saw that around sources of mass $M$ much larger than $M_{P l}$, the effective theory breaks down at much larger distances than the short-distance cutoff scale, parametrically at a radius $\sim\left(M / M_{P l}\right)^{1 / 3} \Lambda^{-1}$.

Of course, the purpose of the effective field theory formalism is not just to understand a single massive graviton. Now that we understand the dynamics of theories with multiple interacting gravitons, we can construct large classes of models with gravity in theory space. As a trivial example, we can consider a theory space version of the first Randall-Sundrum model [30]. We have two sites, one with TeV scale gravity and the standard model and the other with Planck scale gravity. A simple link field will give a TeV mass to one combination of gravitons. At low energies there is a massless graviton with ordinary Planck scale couplings, together with a massive graviton with $1 / \mathrm{TeV}$ couplings to the Standard Model fields. Note that in our set-up there is no need to introduce and stabilize a radion. It would be interesting to extend the theory to more sites in a way that would dynamically generate the large hierarchy of scales in a natural way. Nevertheless, the two-site model has a low quantum gravity cutoff of $\sim \mathrm{TeV}$ which cuts off the Higgs mass quadratic divergence. More generally, we can construct models 
which involve gravitationally sequestered sectors weakly coupled to the standard model. It should also be straightforward to extend our methods to understanding supergravity in theory space. An obvious application of these ideas would be the communication of supersymmetry breaking between sites. For instance we could consider the theory space version of anomaly mediation. It will also be interesting to explore cosmological issues in few site models. In addition to simple constructions with a few sites, we can also consider building gravitational dimensions with many sites, which leads to some fascinating physics that will be discussed in detail in [31].

The most interesting of all possible applications would be the construction of a UV complete theory of gravity. Recall that the deconstruction of non-renormalizable higherdimensional gauge theories has provided them with a UV completion. The structure of the high energy theory was easily guessed at by attempting to UV complete the low-energy non-linear sigma model fields with spontaneous symmetry breaking. Furthermore, with the addition of extra ingredients, such as supersymmetry and conformal invariance, these models lead to deconstructions of non-gravitational sectors of string theory [32]. It is a tantalizing possibility that by pursuing the analogy with the gauge theory which we have begun to develop in this paper, perhaps with some additional ingredients, we may be led to UV completions of four-dimensional quantum gravity.

\section{Acknowledgments}

We would like to thank Andy Cohen and Thomas Gregoire for useful discussions. We understand that M. Luty, M. Porrati, R. Rattazzi and R. Sundrum have been exploring massive gravity in the context of the DGP model [33]. We thank them for discussions. Also, A. Chamseddine has informed us that related ideas can be found in [34]. N.A-H. is supported in part by the Department of Energy under Contracts DE-AC03-76SF00098 and the National Science Foundation under grant PHY-95-14797, the Alfred P. Sloan foundation, and the David and Lucille Packard Foundation.

\section{References}

[1] N. Arkani-Hamed, A. G. Cohen and H. Georgi, Phys. Rev. Lett. 86, 4757 (2001) [arXiv:hep-th/0104005].

[2] C. T. Hill, S. Pokorski and J. Wang, Phys. Rev. D 64, 105005 (2001) [arXiv:hep-th/0104035].

[3] H. C. Cheng, C. T. Hill, S. Pokorski and J. Wang, Phys. Rev. D 64, 065007 (2001) [arXiv:hep-th/0104179].

[4] N. Arkani-Hamed, A. G. Cohen and H. Georgi, JHEP 0207, 020 (2002) [arXiv:hep-th/0109082].

[5] D. G. Boulware and S. Deser, Phys. Lett. B 40, 227 (1972), Phys. Rev. D 63368 (1972).

[6] M. J. Duff, C. N. Pope and K. S. Stelle, Phys. Lett. B 223, 386 (1989).

[7] C. Aragone and S. Deser, Nuovo Cim. A 3, 709 (1971). 
[8] C. R. Nappi and L. Witten, Phys. Rev. D 40, 1095 (1989).

[9] I. L. Buchbinder, D. M. Gitman, V. A. Krykhtin and V. D. Pershin, Nucl. Phys. B 584, 615 (2000) [arXiv:hep-th/9910188].

[10] P. Van Nieuwenhuizen, Nucl. Phys. B 60, 478 (1973).

[11] M. Fierz and W. Pauli, Proc. Roy. Soc. Lond. A 173, 211 (1939).

[12] H. van Dam and M. J. Veltman, Nucl. Phys. B 22, 397 (1970).

[13] V. I. Zakharov, JETP Letters (Sov. Phys.) 12, 312 (1970).

[14] I. I. Kogan, arXiv:astro-ph/0108220.

[15] I. I. Kogan, S. Mouslopoulos, A. Papazoglou and L. Pilo, Nucl. Phys. B 625, 179 (2002) [arXiv:hep-th/0105255].

[16] A. Karch, E. Katz and L. Randall, JHEP 0112, 016 (2001) [arXiv:hep-th/0106261].

[17] M. Porrati, Phys. Lett. B 498, 92 (2001) [arXiv:hep-th/0011152].

[18] I. I. Kogan, S. Mouslopoulos and A. Papazoglou, Phys. Lett. B 503, 173 (2001) [arXiv:hep-th/0011138].

[19] A. I. Vainshtein, Phys. Lett. B 39, 393 (1972).

[20] M. Porrati, Phys. Lett. B 534, 209 (2002) [arXiv:hep-th/0203014].

[21] C. Deffayet, G. R. Dvali, G. Gabadadze and A. I. Vainshtein, Phys. Rev. D 65, 044026 (2002) [arXiv:hep-th/0106001].

[22] A. Lue, Phys. Rev. D 66, 043509 (2002) [arXiv:hep-th/0111168].

[23] S. R. Coleman, J. Wess and B. Zumino, Phys. Rev. 177, 2239 (1969).

[24] C. G. Callan, S. R. Coleman, J. Wess and B. Zumino, Phys. Rev. 177, 2247 (1969).

[25] T. Damour and I. I. Kogan, arXiv:hep-th/0206042.

[26] T. Damour, I. I. Kogan and A. Papazoglou, arXiv:hep-th/0206044.

[27] R. P. Feynman, F. B. Morinigo, W. G. Wagner and B. . Hatfield, Reading, USA: Addison-Wesley (1995) $232 \mathrm{p}$. (The advanced book program).

[28] W. Siegel, Phys. Rev. D 49, 4144 (1994) [arXiv:hep-th/9312117].

[29] M. B. Green and C. B. Thorn, Nucl. Phys. B 367, 462 (1991).

[30] L. Randall and R. Sundrum, Phys. Rev. Lett. 83, 3370 (1999) [arXiv:hep-ph/9905221].

[31] N. Arkani-Haned and M. D. Schwartz, work in preparation.

[32] N. Arkani-Hamed, A. G. Cohen, D. B. Kaplan, A. Karch and L. Motl; hep-th/0110146.

[33] G. R. Dvali, G. Gabadadze and M. Porrati, Phys. Lett. B 485, 208 (2000) [arXiv:hep-th/0005016].

[34] A. H. Chamseddine, A. Salam and J. Strathdee, IC-77-148. 